\title{
Independent risk factors of hypoxemia in patients after surgery with acute type $A$ aortic dissection
}

\author{
Jing Zhou ${ }^{1,2,3 \#}$, Jieyi Pan ${ }^{1,2,3 \#}$, Yuheng Yu ${ }^{1,2,3 \#}$, Weixiang Huang ${ }^{1,2,3}$, Yan Lai ${ }^{1,2,3}$, Weibo Liang ${ }^{1,2,3}$, \\ Lingbo Nong ${ }^{1,2,3}$, Xuesong Liu ${ }^{1,2,3}$, Sibei Chen ${ }^{1,2,3}$, Yonghao Xu ${ }^{1,2,3}$, Weiqun $\mathrm{He}^{1,2,3}$, Yuanda Xu ${ }^{1,2,3}$, \\ Xiaoqing Liu ${ }^{1,2,3}$, Yimin Li $^{1,2,3}$, Yongbo Huang ${ }^{1,2,3}$, Ling Sang ${ }^{1,2,3,4}$
}

${ }^{1}$ Department of Critical Care Medicine, The First Affiliated Hospital of Guangzhou Medical University, Guangzhou, China; ${ }^{2}$ Guangzhou Institute of Respiratory Health, Guangzhou, China; ${ }^{3}$ State Key Laboratory of Respiratory Disease, Guangzhou, China; ${ }^{4}$ Guangzhou Laboratory, Guangzhou, China

Contributions: (I) Conception and design: J Zhou, Y Huang, L Sang; (II) Administrative support: X Liu, Y Li, Y Huang, L Sang; (III) Provision of study materials or patients: J Zhou, Y Yu, L Nong, W Liang, X Liu, Y Xu, S Chen, W He, Y Xu; (IV) Collection and assembly of data: J Pan, W Huang; (V) Data analysis and interpretation: Y Lai, L Sang; (VI) Manuscript writing: All authors; (VII) Final approval of manuscript: All authors.

\#These authors contributed equally to this work.

Correspondence to: Ling Sang; Yongbo Huang. State Key Laboratory of Respiratory Diseases; Guangzhou Institute of Respiratory Health; The First Affiliated Hospital of Guangzhou Medical University, 151 Yanjiang Street West, Guangzhou 510120, China.

Email: sonysang999@vip.163.com; yongbo2046@163.com.

Background: This study aimed to investigate independent risk factors of postoperative hypoxemia in patients with acute type A aortic dissection (ATAAD).

Methods: A single-center retrospective study was conducted with enrolled 75 ATAAD patients following surgery, which were stratified into three groups on the basis of the postoperative $\mathrm{PaO}_{2} / \mathrm{FiO}_{2}$ ratio: severe hypoxemia group $\left(\mathrm{PaO}_{2} / \mathrm{FiO}_{2}\right.$ ratio $\left.\leq 100 \mathrm{mmHg}\right)$; moderate hypoxemia group $\left(100 \mathrm{mmHg}<\mathrm{PaO}_{2} / \mathrm{FiO}_{2}\right.$ ratio $\leq 200 \mathrm{mmHg})$; and non-hypoxemia group $\left(\mathrm{PaO}_{2} / \mathrm{FiO}_{2}\right.$ ratio $\left.>200 \mathrm{mmHg}\right)$. The patient's demography, perioperative laboratory results, operative details, clinical outcomes were collected and analyzed. Univariable and multivariable analyses were performed and logistic regression model was established.

Results: The incidence of postoperative severe hypoxemia and hypoxemia was $32 \%$ and $52 \%$, respectively. Among the three groups, severe hypoxemia group exhibited a high significance of body mass index (BMI) and preoperative white blood cell (WBC) and main distribution of hypertension; meanwhile, Marfan syndrome was mainly distributed in non-hypoxemia group. On intensive care unit (ICU) admission, severe hypoxemia group exhibited a high significance of Acute Physiology and Chronic Health Evaluation (APACHE II) score of postoperative patients, and more patients would present shock. Moreover, severe hypoxemia group patients had a higher incidence of postoperative acute kidney injury (AKI) and usage of renal replacement therapy, longer length of stay (LOS) of ICU, and shorter 28 days ventilator-free days (VFDs).

Conclusions: The incidence of postoperative hypoxemia was high in ATAAD patients owing to comprehensive high-risk factors. Besides, postoperative complications negatively impacted their clinical outcomes.

Keywords: Acute type A aortic dissection (ATAAD); postoperative; hypoxemia; risk factor

Submitted May 30, 2021. Accepted for publication Jun 18, 2021.

doi: 10.21037/apm-21-1428

View this article at: https://dx.doi.org/10.21037/apm-21-1428 


\section{Introduction}

Acute type A aortic dissection (ATAAD) is a life-threatening condition associated with severe morbidity and mortality (1) and risk factors for AAD have been evaluated, such as gender, age, hypertension, smoking, aneurysm, congenital disorders, and inflammatory disease (2). Despite improved surgical technical and perioperative management, the incidence of postoperative complications remains high (3). Postoperative hypoxemia, one of the most common complications, is followed by prolonged mechanical ventilation duration, increased length of stay (LOS) of intensive care unit (ICU), even increased mortality. For postoperative severe postoperative hypoxemia $\left(\mathrm{PaO}_{2} /\right.$ $\mathrm{FiO}_{2}$ ratio $<100 \mathrm{mmHg}$ ) of ATAAD, even rescue therapy is required, such as prone position ventilation (PPV) and extracorporeal membrane oxygenation (ECMO) $(4,5)$. Therefore, it is crucial to identify patients who will develop such a critical condition, making medical resource assignments more reasonable and improving the prognosis for such a population.

Several risk factors of postoperative hypoxemia for ATAAD patients, such as obesity, female, preoperative $\mathrm{PaO}_{2} / \mathrm{FiO}_{2}$ ratio $\leq 300 \mathrm{mmHg}$, blood transfusion, have been investigated in some studies; however, the results remain controversial (6-12). Through retrospective analysis of patients' data, to determine the risk factors of hypoxemia and its severity can help clinicians to adjust treatment strategies and avoid the occurrence of hypoxemia. As mentioned above, we conducted a retrospective study to assess the incidence, risk factors, as well as impact on clinical outcome of postoperative hypoxemia and severe postoperative hypoxemia of ATAAD. We present the following article in accordance with the SROBE reporting checklist (available at https://dx.doi.org/10.21037/apm21-1428).

\section{Methods}

\section{Study design}

A single-center retrospective study was conducted in a 37 beds general ICU of the $1^{\text {st }}$ affiliated hospital of Guangzhou Medical University. From January 2016 to May 2020, the patients diagnosed as ATAAD confirmed by enhanced computed tomographic scan and underwent urgent operation were included. However, patients were excluded if they died during operation or within 24 hours after surgery. The study was conducted as per the Declaration of Helsinki (as revised in 2013). The study was granted approval by the ethics committee at The $1^{\text {st }}$ affiliated hospital of Guangzhou Medical University (Medical research ethics review: 2021NO. K-12), and individual consent for this retrospective analysis was waived.

\section{Data collection}

Overall, 75 subjects were enrolled, and data were extracted from our electronic hospital information system (HIS). The patient's demography [age, gender, body mass index (BMI), and comorbidities], perioperative laboratory results, operative details (operation time, CPB time, clamping time, intraoperative blood and plasma transfusions), postoperative $\mathrm{P} / \mathrm{F}$ rate (the worst value from blood gas analysis within 72 hours after ICU admission), and clinical outcomes [28 days ventilator-free days (VFD), mechanical ventilation time, LOS of ICU, LOS of hospital, occurrence of acute kidney injury (AKI) and mortality] were collected and analyzed. We divided the patients into three groups according to postoperative $\mathrm{PaO}_{2} / \mathrm{FiO}_{2}$ ratio, selecting the worst condition according to blood gas analysis within 72 hours after surgery: group severe (severe hypoxemia group, $\mathrm{PaO}_{2} / \mathrm{FiO}_{2}$ ratio $\leq 100 \mathrm{mmHg}$ ); group moderate (moderate hypoxemia group, $100 \mathrm{mmHg}<\mathrm{PaO}_{2} / \mathrm{FiO}_{2}$ ratio $\leq 200 \mathrm{mmHg}$ ); and group mild (non-hypoxemia group, $\mathrm{PaO}_{2} / \mathrm{FiO}_{2}$ ratio $>200 \mathrm{mmHg}$ ).

\section{Surgical procedure}

After standard anaesthetic management, patients were provided a total arch replacement and descending aorta stent implantation which was conducted according to the Sun's produce (13). Briefly, a median sternotomy procedure was used in all the operations. For the arterial perfusion, routine exposure of the right axillary artery was done, and for venous drainage, cannulation of the inferior of superior vena cava or the right atrium was performed. The patients were then heparinized, followed by establishment of the cardiopulmonary bypass (CPB). When the CPB started, we initiated systemic cooling. Clamping of the ascending aorta was done, followed by longitudinal opening, and subsequent infusion of cardioplegic solution consisting of cold-blood into the right and left coronary artery separately. Surgery of the aortic root, entailing David or Bentall surgery or replacement of the ascending aorta was done based on the lesions of the aortic root during the cooling process. At a temperature of between $17{ }^{\circ} \mathrm{C}$ and $26^{\circ} \mathrm{C}$ 
in the nasopharynx, and $19{ }^{\circ} \mathrm{C}$ and $29^{\circ} \mathrm{C}$ in the rectum, the brachiocephalic artery, left common carotid artery, as well as left subclavian artery were sequentially clamped, followed by initiation of the DHCA (deep hypothermic circulatory arrest) along with SCP (selective cerebral perfusion) via the right axillary artery $(5-10 \mathrm{~mL} / \mathrm{kg})$. Transection of the aortic arch was done between the left subclavian artery and the left common carotid artery. After that, a stented elephant graft (MicroPort Medical Co. Ltd., China) was first released in the descending aorta. Direct saturation of the left subclavian artery residual in the arch was performed. Thereafter, the three parts consisting of the stented graft, four-branch prosthetic graft, and the descending aorta were anastomosised, followed by immediate lower body reperfusion via perfusion with half of the normal flow in one limb of the four-branch prosthetic graft. Bilateral perfusion of the brain was done at the end of the anastomosis action on the left common carotid artery. Thereafter, normal flow was resumed in the CPB gradually and began to rewarm, then saturation of the bleeding was done on the distal anastomosis. Resuscitation of the heart was done after the area between the proximal aortic arch and the ascending aorta was anastomosed. Afterwards, we separately anastomosed the brachiocephalic artery along with the left subclavian artery and the respective branch of the four-branch prosthetic graft. Finally, drainage was made to the right atrium with the arterial wall residual or the pericardium or biological patch about the artificial graft. After completion of the repair and adequate rewarming, the patient was weaned from CBP.

\section{Management after surgery}

After surgery, the patients were placed in ICU and received comprehensive care according to our protocol: volumecontrolled ventilation was performed in the ICU with a minimum $\mathrm{FiO}_{2}$ and a PEEP (positive end expiratory pressure) of 6-10 $\mathrm{cmH}_{2} \mathrm{O}$ to achieve $\mathrm{SpO}_{2} 95-97 \%$. For patients who suffered from respiratory distress or moderate to severe hypoxemia $(\mathrm{P} / \mathrm{F}<150 \mathrm{mmHg}$, deep sedation or even neuromuscular block (Richmond agitation sedation scale, RASS -5) and protective ventilatory strategy were performed. If the condition was not improved, prone positioning ventilation, even ECMO was applied to the patients. Furthermore, renal replacement therapy (RRT) was used to the patients who suffered from oliguria caused by $\mathrm{AKI}$ and required liquid management.

\section{Statistical analysis}

Descriptive data are given as mean, ranges, standard deviation, and median for continuous variables and as percentages and counts for categorical variables. A $\chi^{2}$ test with Fisher's exact test or ANOVA test was used to compare groups as appropriate. A two-tailed $\mathrm{P}$ value less than 0.05 was considered statistically significant. Variables with a $\mathrm{P}$ value less than 0.1 or less at univariate analysis were included in a logistic regression multivariate analysis with a stepwise forward conditional approach, estimates of odds ratio (OR), 95\% CI and $\mathrm{P}$ values are displayed. All analyses were implemented in SPSS V. 20 (IBM Corp, Armonk, $\mathrm{NY}$ ). All $\mathrm{P}$ values are based on 2 -sided tests and considered statistically significant at $\mathrm{P}<0.05$.

\section{Results}

\section{Baseline characteristics}

A total of 75 enrolled patients, comprising 61 males (81.3\%), with a mean age of $(53.53 \pm 13.98)$ years, were divided into three groups: group severe (24 patients), group moderate (39 patients), and group mild (12 patients). The incidence of postoperative severe hypoxemia and hypoxemia was $32 \%$ and $52 \%$, respectively, and only $16 \%$ of patients did not develop such complications. Among the three groups, BMI and preoperative white blood cell (WBC) were remarkably higher in group severe $(26.20 \pm 3.40$ vs. $22.98 \pm 4.94$ vs. $20.75 \pm 4.03 \mathrm{~kg} / \mathrm{m}^{2}, \mathrm{P}=0.001$, and $12.48 \pm 3.97$ vs. $10.5 \pm 3.80$ vs. $8.69 \pm 2.48 \mathrm{~kg} / \mathrm{m}^{2}, \mathrm{P}=0.014$, respectively). Hypertension was mainly distributed in group severe $(91.67 \%$ vs. $82.05 \%$ vs. $50 \%, \mathrm{P}=0.015)$. Meanwhile, Marfan syndrome was mainly distributed in group mild ( $0 \%$ vs. $2.56 \%$ vs. $25 \%$, $\mathrm{P}=0.01$ ). The other variables, such as age, gender, medical history, and preoperative laboratory test results, were not remarkably different. More details are shown in Table 1.

\section{Intraoperative and postoperative characteristics of patients}

The intraoperative details were similar among the three groups. The Acute Physiology and Chronic Health Evaluation (APACHE II) score of postoperative patients on ICU admission was remarkably greater in group severe (21.75 \pm 6.27 vs. $18.18 \pm 7.38$ vs. $15.75 \pm 6.00, \mathrm{P}=0.033)$, and more patients would present shock on ICU admission in group severe ( $37.5 \%$ vs. $28.2 \%$ vs. $0 \%, \mathrm{P}=0.042)$. The other postoperative patient's characteristics and laboratory test 
Table 1 Characteristics of patients with acute type A aortic dissection

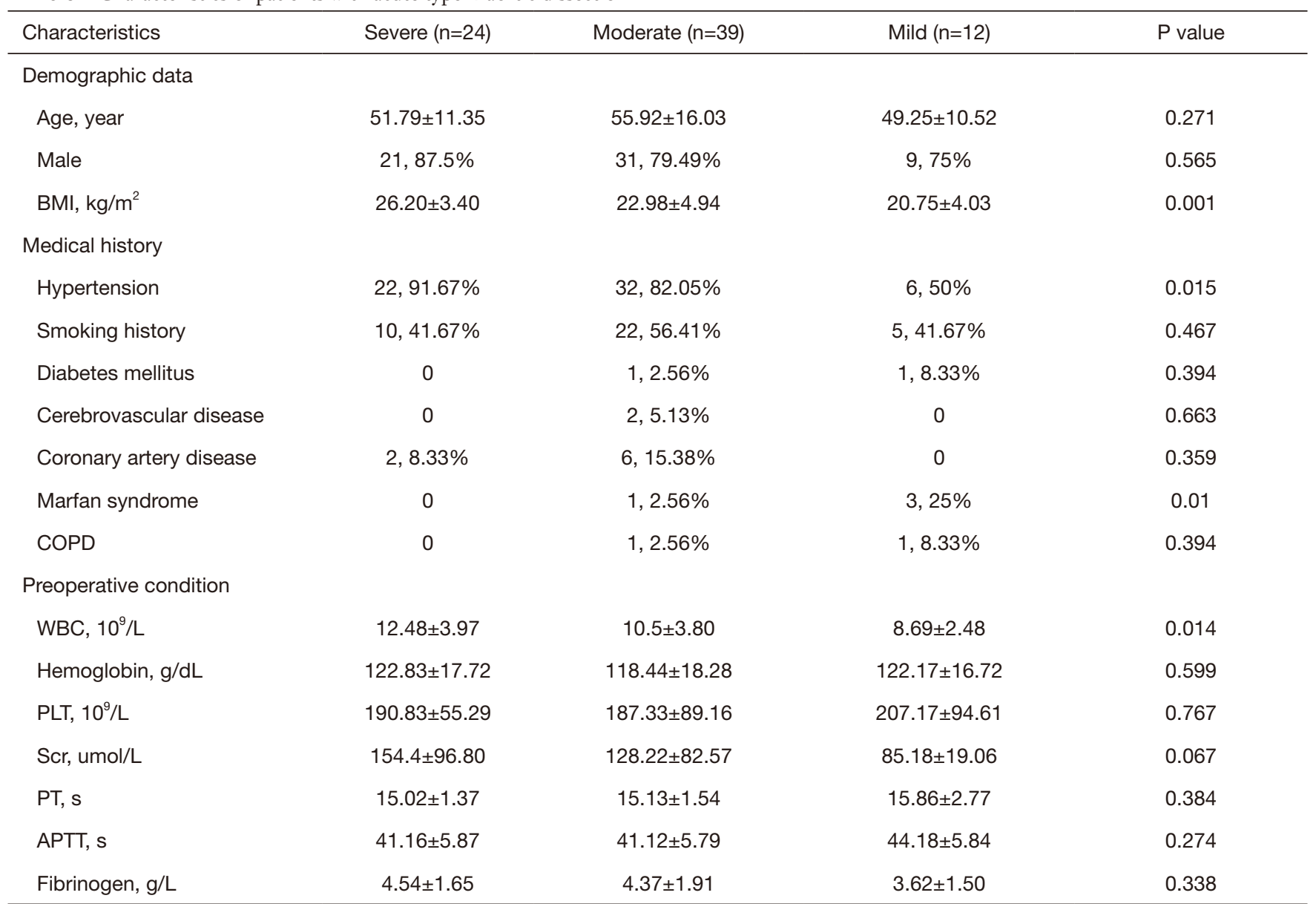

Values are presented as mean \pm standard deviation or ( $\mathrm{n}, \%)$. BMI, body mass index; COPD, chronic obstructive pulmonary disease; WBC, white blood cell; PLT, platelet; PT, prothrombin time; APTT, activated partial prothrombin time; Scr, serum creatinine.

results were similar among the three groups. More details are presented in Table 2 .

\section{Clinical outcomes}

The subjects in group severe had a higher incidence of postoperative AKI and usage of renal replacement therapy, longer LOS of ICU, and shorter 28 days VFDs $(\mathrm{P}=0.002$, 0.027 , and 0.043 , respectively). Notably, though there was no remarkable difference in mortality among the three groups, group mild patients were all survived. More details are displayed in Table 3.

\section{Risk factors for postoperative hypoxemia, severe hypoxemia and the prediction models}

In the first step, we compared group (severe and moderate) with group mild to evaluate the risk factor for postoperative hypoxemia $\left(\mathrm{PaO}_{2} / \mathrm{FiO}_{2}\right.$ ratio $\left.\leq 200 \mathrm{mmHg}\right)$. In the univariate analysis, $\mathrm{BMI}$, preoperative $\mathrm{WBC}$, preoperative serum creatinine, and intraoperative $\mathrm{CPB}$ time were remarkably higher in group (severe and moderate) $(\mathrm{P}=0.019,0.035$, 0.047 , and 0.024 , respectively). Hypertension and postoperative shock were mainly distributed in group (severe and moderate) $(\mathrm{P}=0.011$ and 0.029 , respectively); meanwhile, Marfan syndrome was mainly distributed in group mild ( $\mathrm{P}=0.012)$. Although postoperative APACHE II score was not remarkably higher in group (severe and moderate) $(\mathrm{P}=0.089)$, we included this variable into the logistic regression according to our predefined statistical analysis protocol. We found that only hypertension was (OR 0.117, 95\% CI: 0.023-0.589, $\mathrm{P}=0.009$ ) independent risk factors using logistic regression. More details are presented in Tables 4 and 5. 
Table 2 The intraoperative and postoperative characteristics of patients

\begin{tabular}{|c|c|c|c|c|}
\hline & Severe $(n=24)$ & Moderate $(n=39)$ & Mild $(n=12)$ & $P$ value \\
\hline The duration of operation, hour & $8.60 \pm 2.01$ & $8.58 \pm 1.93$ & $7.76 \pm 1.24$ & 0.390 \\
\hline Aortic clamping time, $\min$ & $148 \pm 36.18$ & $145.77 \pm 32.66$ & $131.67 \pm 40.2$ & 0.408 \\
\hline CPB time, min & $259.38 \pm 66.23$ & $246 \pm 66.04$ & $204.17 \pm 49.64$ & 0.058 \\
\hline RBCs transfusion, $\mathrm{u}$ & $4.65 \pm 3.59$ & $4.5 \pm 4.64$ & $3.79 \pm 2.14$ & 0.831 \\
\hline PLTs transfusion, $u$ & $1.04 \pm 0.68$ & $1.05 \pm 0.68$ & $0.75 \pm 0.43$ & 0.360 \\
\hline Intraoperative blood loss, mL & $1,564.79 \pm 1,339.74$ & $1,477.18 \pm 932.72$ & $1,300.0 \pm 1,055.75$ & 0.800 \\
\hline \multicolumn{5}{|l|}{ Postoperative condition } \\
\hline WBC, $10^{9} / \mathrm{L}$ & $20.10 \pm 7.88$ & $19.18 \pm 5.63$ & $19.45 \pm 6.01$ & 0.867 \\
\hline Shock & $9,37.5 \%$ & $11,28.2 \%$ & 0 & 0.042 \\
\hline Cardiogenic shock & $4,16.67 \%$ & $8,20.51 \%$ & 0 & 0.267 \\
\hline Obstructive shock & 0 & 0 & 0 & NS \\
\hline Septic shock & $5,20.83 \%$ & $2,5.13 \%$ & 0 & 0.083 \\
\hline Hemorrhagic shock & $3,12.5 \%$ & $5,12.82 \%$ & 0 & 0.605 \\
\hline APACHE II & $21.75 \pm 6.27$ & $18.18 \pm 7.38$ & $15.75 \pm 6.00$ & 0.033 \\
\hline SOFA & $11.33 \pm 3.48$ & $9.95 \pm 3.91$ & $9.33 \pm 3.03$ & 0.217 \\
\hline
\end{tabular}

Values are presented as mean \pm standard deviation or $(n, \%)$. CPB, cardiopulmonary bypass; RBCs, red blood cells; WBC, white blood cell, PLT, platelet, PCT, procalcitonin; APACHE II, acute physiology and chronic health evaluation; SOFA, sequential organ failure assessment.

Table 3 Patients' clinical outcome

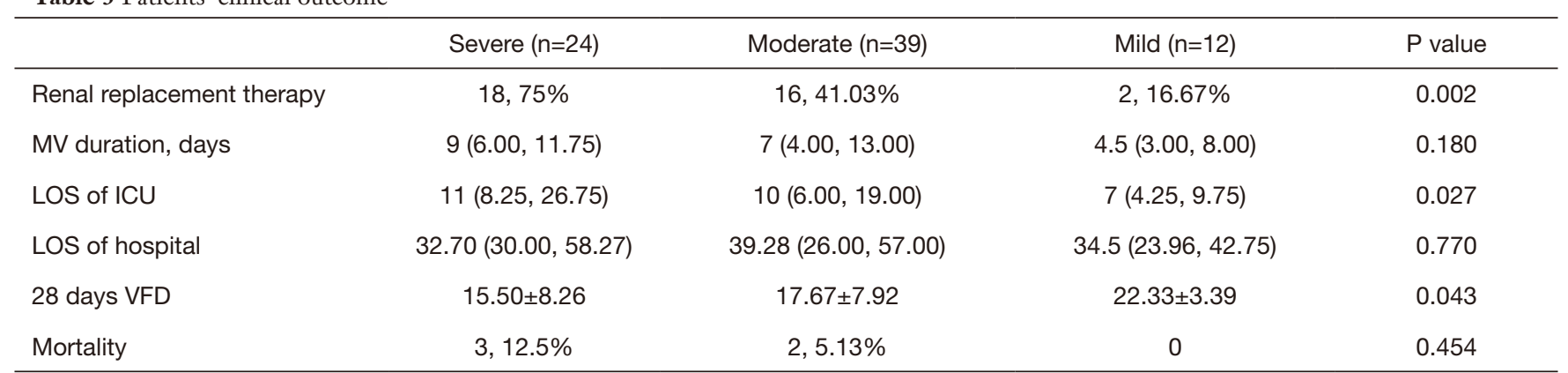

Values are presented as mean \pm standard deviation, (n, \%), or median (interquartile range). MV, mechanical ventilation; LOS, length of stay; 28 days VFD, numbers of ventilator-free days at 28 days.

In the second step, we used the same strategy to compare group severe with group (moderate and mild) to evaluate the risk factor for severe postoperative hypoxemia $\left(\mathrm{PaO}_{2} /\right.$ $\mathrm{FiO}_{2}$ ratio $\leq 100 \mathrm{mmHg}$ ). In the univariate analysis, BMI, preoperative $\mathrm{WBC}$, and postoperative APACHE II score were remarkably higher in group severe $(\mathrm{P}=0.001,0.011$ and 0.017 , respectively). Although preoperative serum creatinine, postoperative PCT, and postoperative SOFA 
Table 4 Univariate analysis of postoperative hypoxemia risk factors (severe and moderate groups vs. mild group)

\begin{tabular}{|c|c|c|c|}
\hline Characteristics & Severe and moderate $(n=63)$ & Mild $(n=12)$ & $P$ value \\
\hline \multicolumn{4}{|l|}{ Demographic data } \\
\hline Age, year & $54.35 \pm 14.47$ & $49.25 \pm 10.52$ & 0.249 \\
\hline Male & $52,82.5 \%$ & $9,75 \%$ & 0.686 \\
\hline $\mathrm{BMI}, \mathrm{kg} / \mathrm{m}^{2}$ & $24.20 \pm 4.66$ & $20.75 \pm 4.03$ & 0.019 \\
\hline Hypertension & $54,85.7 \%$ & $6,50 \%$ & 0.011 \\
\hline Smoking history & $32,50.8 \%$ & $5,41.67 \%$ & 0.754 \\
\hline Diabetes mellitus & $1,1.6 \%$ & $1,8.33 \%$ & 0.296 \\
\hline Cerebrovascular disease & $2,3.2 \%$ & 0 & 1.0 \\
\hline COPD & $1,1.6 \%$ & $1,8.33 \%$ & 0.296 \\
\hline \multicolumn{4}{|l|}{ Preoperative condition } \\
\hline WBC, $10^{9} / \mathrm{L}$ & $11.25 \pm 3.95$ & $8.69 \pm 2.48$ & 0.035 \\
\hline Hemoglobin, g/dL & $120.11 \pm 18.05$ & $122.17 \pm 16.72$ & 0.716 \\
\hline $\mathrm{PLT}, 10^{\circ} / \mathrm{L}$ & $188.67 \pm 78.65$ & $207.17 \pm 94.61$ & 0.476 \\
\hline Scr, umol/L & $138.2 \pm 89.89$ & $85.18 \pm 19.06$ & 0.047 \\
\hline PT, s & $15.08 \pm 1.49$ & $15.86 \pm 2.77$ & 0.171 \\
\hline APTT, s & $41.13 \pm 5.86$ & $44.18 \pm 5.84$ & 0.106 \\
\hline Plasma transfusion, $\mathrm{mL}$ & $760.3 \pm 434.14$ & $587.5 \pm 150.17$ & 0.179 \\
\hline RBCs transfusion, $\mathrm{u}$ & $4.56 \pm 4.30$ & $3.79 \pm 2.14$ & 0.552 \\
\hline PLTs transfusion, $u$ & $1.05 \pm 0.68$ & $0.75 \pm 0.43$ & 0.152 \\
\hline Intraoperative blood loss, mL & $1,510.6 \pm 1,115.29$ & $1,300.0 \pm 1,055.75$ & 0.550 \\
\hline \multicolumn{4}{|l|}{ Postoperative condition } \\
\hline $\mathrm{PCT}, \mathrm{ng} / \mathrm{mL}$ & $34.57 \pm 45.4$ & $16.94 \pm 11.9$ & 0.188 \\
\hline WBC, $10^{\circ} / \mathrm{L}$ & $19.53 \pm 6.65$ & $19.45 \pm 6.01$ & 0.968 \\
\hline Shock & $20,31.7 \%$ & 0 & 0.029 \\
\hline APACHE II & $19.54 \pm 7.14$ & $15.75 \pm 6.00$ & 0.089 \\
\hline SOFA & $10.48 \pm 3.78$ & $9.33 \pm 3.03$ & 0.327 \\
\hline
\end{tabular}

Values are presented as mean \pm standard deviation or ( $\mathrm{n}, \%)$. BMl, body mass index; COPD, chronic obstructive pulmonary disease; WBC, white blood cell; PLT, platelet; PT, prothrombin time; APTT, activated partial prothrombin time; Scr, serum creatinine; CPB, cardiopulmonary bypass; RBCs, red blood cells; PCT, procalcitonin; APACHE II, acute physiology and chronic health evaluation; SOFA, sequential organ failure assessment. 
Table 5 Risk factors of postoperative hypoxemia (severe and moderate groups vs. mild group) in multivariate logistic regression analysis

\begin{tabular}{lccc}
\hline Clinical variables & OR & $95 \% \mathrm{Cl}$ & $\mathrm{P}$ value \\
\hline Hypertension & 0.117 & $0.023-0.589$ & 0.009 \\
\hline
\end{tabular}

$\mathrm{Cl}$, confidence interval; OR, odds ratio.

score were not remarkably higher in group severe $(\mathrm{P}=0.084$, 0.086 , and 0.093 , respectively), we included these variables in the logistic regression as mentioned above. Using logistic regression, we found that BMI (OR 0.758, 95\% CI: 0.640$0.897, \mathrm{P}=0.001)$ and postoperative APACHE II score (OR 0.900, 95\% CI: $0.828-0.978, \mathrm{P}=0.013$ ) were independent risk factors. More details are shown in Tables 6 and 7.

\section{Discussion}

Hypoxemia is a common postoperative complication in ATAAD patients. Our study illustrated that the incidence of such complication was $84 \%(63 / 75)$, much higher than previous studies (30-50\%) (6-11). However, the incidence of severe hypoxemia $(\mathrm{P} / \mathrm{F}<100 \mathrm{mmHg})$ was $32 \%(24 / 75)$ in our study, slightly lower than a recent study (36.6\%) (12). We reasoned that since most of our patients transported from other hospitals takes a long time from symptom onset to entering the operating room, which would put the patients at a higher risk to develop the postoperative hypoxemia.

Herein, we illustrated that higher BMI was linked to developing postoperative hypoxemia and severe hypoxemia in line with previous studies (8-12). Obesity, defined as BMI $>25 \mathrm{~kg} / \mathrm{m}^{2}$, had significant adverse effects on cardiopulmonary physiology. Obese patients often showed a decreased functional residual capacity (FRC), total lung capacity (TLC), expiratory reserve volume (ERV), as well as vital capacity (VC), and severity was inversely proportional to BMI. In addition, obesity could lead to abdominal distention, resulting in decreased chest wall compliance, increased respiratory resistance, pleural pressure, and upper and lower airway resistance (14). Besides, obesity was associated with chronic increased proinflammatory cytokines and mediators, leading to cell membrane damage (15). Consequently, obesity was considered as an independent high-risk factor of postoperative pulmonary complications, and improved intra and postoperative ventilation strategies should be crucial in such populations $(16,17)$.

The mechanical and physical effects, such as inflammatory cascade reaction and pulmonary ischemia-reperfusion injury, caused by CPB were considered a significant cause of postoperative hypoxemia $(10,12)$. Our result showed that $\mathrm{CPB}$ time was longer in severe and moderate groups $(\mathrm{P} / \mathrm{F}$ ratio $\leq 200 \mathrm{mmHg}$ ) in contrast with the mild group (P/ F ratio $>200 \mathrm{mmHg}$ ).

Elevated APACHE II score and occurrence of postoperative shock were identified to contribute to postoperative hypoxemia development in our study. Given that these indexes were developed to represent disease's seriousness, it was unsurprising to discover this connection. It worth noting that both hypertension and Marfan syndrome were recognized as risk factors of AAD. Our study illustrated that hypertension was associated with postoperative hypoxemia development; however, Marfan syndrome seemed to be a protective factor. Also, hypertension was mainly distributed in groups severe and group moderate, and Marfan syndrome was all in group mild. It seemed that if AAD was induced by Marfan syndrome rather than hypertension, it might develop postoperative hypoxemia.

Our study showed that severe postoperative hypoxemia would negatively impact clinical outcomes, including higher RRT usage, longer LOS of ICU, and shorter 28 days VFDs. The incidence of AKI, which manifests through rapid loss of renal function with high morbidity and mortality (18), remained high in such a population $(19,20)$. The postoperative low oxygen supply and high oxygen might play a vital role in this progression (21). Interestingly, the linear relationship between obesity and AKI was illustrated (22) as an essential contributor to postoperative hypoxemia.

There were two limitations in our study. First, it was a single-center retrospective observational study, and the sample size was limited. Secondly, the impact of factors, including individual surgeon's experience along with institutional philosophy with regards to treatment decision was not considered in this analysis.

\section{Conclusions}

Herein, we established that postoperative moderate and severe hypoxemia were high in ATAAD patients. The 
Table 6 Univariate analysis of the risk factors of postoperative hypoxemia (moderate and mild groups vs. severe group)

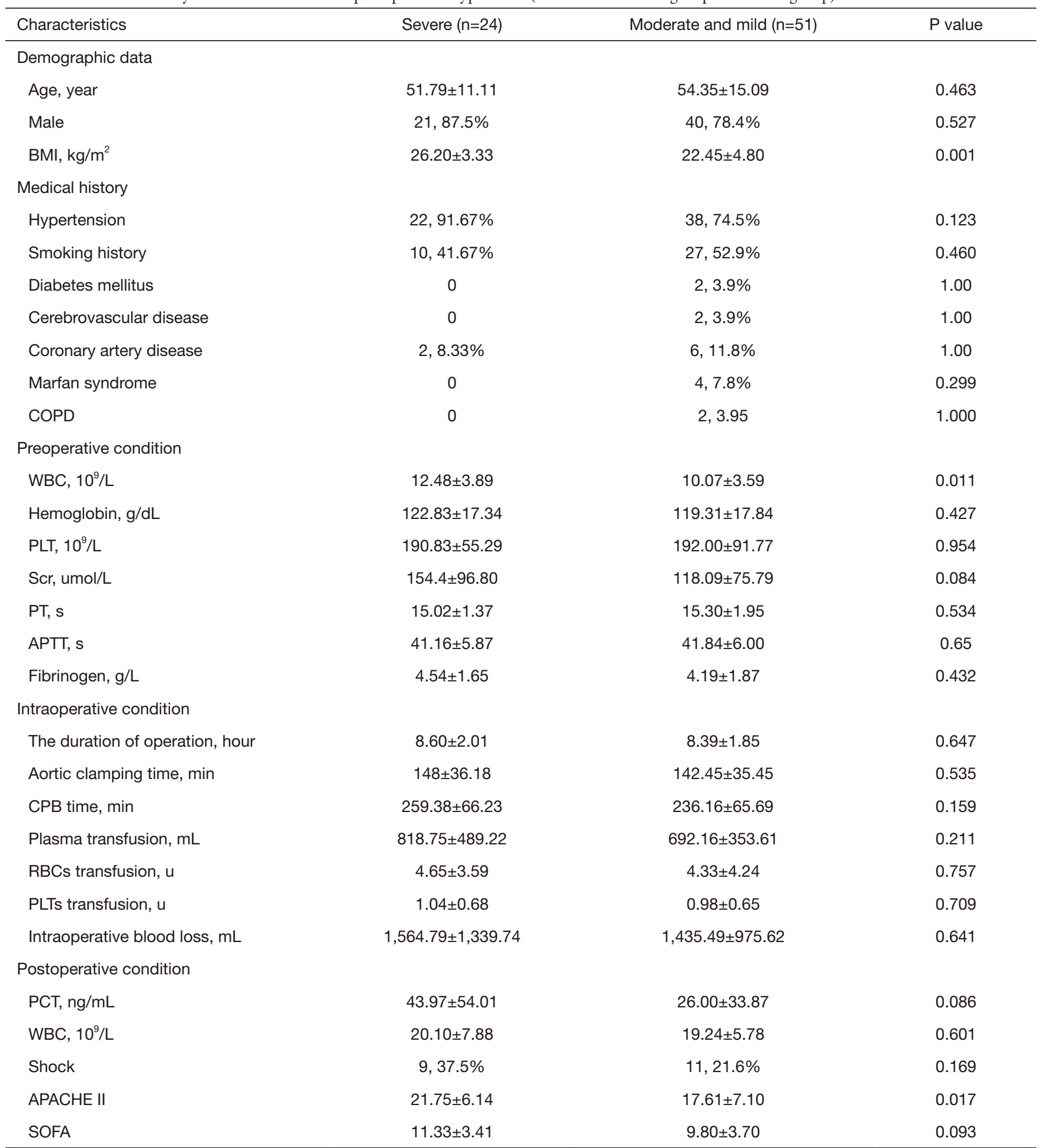

Values are presented as mean \pm standard deviation or $(n, \%)$. BMI, body mass index; COPD, chronic obstructive pulmonary disease; WBC, white blood cell; PLT, platelet; PT, prothrombin time; APTT, activated partial prothrombin time; Scr, serum creatinine; CPB, cardiopulmonary bypass; RBCs, red blood cells; PCT, procalcitonin; APACHE II, acute physiology and chronic health evaluation; SOFA, sequential organ failure assessment. 
Table 7 Risk factors of postoperative severe hypoxemia (moderate and mild groups $v s$. severe group) in multivariate logistic regression analysis

\begin{tabular}{lccc}
\hline Clinical variables & OR & $95 \% \mathrm{Cl}$ & P value \\
\hline BMI & 0.758 & $0.640-0.897$ & 0.001 \\
APACHE II & 0.900 & $0.828-0.978$ & 0.013 \\
\hline
\end{tabular}

BMI, body mass index; APACHE II, acute physiology and chronic health evaluation.

high-risk factors had been evaluated. Meanwhile, such postoperative complications negatively impacted the clinical outcomes.

\section{Acknowledgments}

Funding: This study was supported by Emergency Key Program of Guangzhou Laboratory (Grant No. EKPG2117), The Special Project of Guangdong Science and Technology Department (2020B1111340016) and Natural Science Foundation of Guangdong Province (Grant No. 2020A1515011459).

\section{Footnote}

Reporting Checklist: The authors have completed the STROBE reporting checklist. Available at https://dx.doi. org/10.21037/apm-21-1428

Data Sharing Statement: Available at https://dx.doi. org/10.21037/apm-21-1428

Conflicts of Interest: All authors have completed the ICMJE uniform disclosure form (available at https://dx.doi. org/10.21037/apm-21-1428). The authors have no conflicts of interest to declare.

Ethical Statement: The authors are accountable for all aspects of the work in ensuring that questions related to the accuracy or integrity of any part of the work are appropriately investigated and resolved. The study was conducted in accordance with the Declaration of Helsinki (as revised in 2013). The study was approved by the Ethics Committee at the First Affiliated Hospital of Guangzhou Medical University (Medical research ethics review: 2021NO. K-12), and individual consent for this retrospective analysis was waived.

Open Access Statement: This is an Open Access article distributed in accordance with the Creative Commons
Attribution-NonCommercial-NoDerivs 4.0 International License (CC BY-NC-ND 4.0), which permits the noncommercial replication and distribution of the article with the strict proviso that no changes or edits are made and the original work is properly cited (including links to both the formal publication through the relevant DOI and the license). See: https://creativecommons.org/licenses/by-nc-nd/4.0/.

\section{References}

1. Jassar AS, Sundt TM 3rd. How should we manage type A aortic dissection? Gen Thorac Cardiovasc Surg 2019;67:137-45.

2. Gawinecka J, Schönrath F, von Eckardstein A. Acute aortic dissection: pathogenesis, risk factors and diagnosis. Swiss Med Wkly 2017;147:w14489.

3. Gudbjartsson T, Ahlsson A, Geirsson A, et al. Acute type A aortic dissection - a review. Scand Cardiovasc J 2020;54:1-13.

4. Girdauskas E, Kuntze T, Borger MA, et al. Acute respiratory dysfunction after surgery for acute type A aortic dissection. Eur J Cardiothorac Surg 2010;37:691-6.

5. Yokota K, Fujii T, Kimura K, et al. Life-threatening hypoxemic respiratory failure after repair of acute type a aortic dissection: successful treatment with venoarterial extracorporeal life support using a prosthetic graft attached to the right axillary artery. Anesth Analg 2001;92:872-6.

6. Shen Y, Liu C, Fang C, et al. Oxygenation impairment after total arch replacement with a stented elephant trunk for type-A dissection. J Thorac Cardiovasc Surg 2018;155:2267-74.

7. Gao Z, Pei X, He C, et al. Oxygenation impairment in patients with acute aortic dissection is associated with disorders of coagulation and fibrinolysis: a prospective observational study. J Thorac Dis 2019;11:1190-201.

8. Liu N, Zhang W, Ma W, et al. Risk factors for hypoxemia following surgical repair of acute type A aortic dissection. Interact Cardiovasc Thorac Surg 2017;24:251-6.

9. Wang Y, Xue S, Zhu H. Risk factors for postoperative hypoxemia in patients undergoing Stanford A aortic dissection surgery. J Cardiothorac Surg 2013;8:118. 
10. Nakajima T, Kawazoe K, Izumoto H, et al. Risk factors for hypoxemia after surgery for acute type A aortic dissection. Surg Today 2006;36:680-5.

11. Ge H, Jiang Y, Jin Q, et al. Nomogram for the prediction of postoperative hypoxemia in patients with acute aortic dissection. BMC Anesthesiol 2018;18:146.

12. Gong $M, W u Z, X u S$, et al. Increased risk for the development of postoperative severe hypoxemia in obese women with acute type a aortic dissection. J Cardiothorac Surg 2019;14:81.

13. Sun LZ, Ma WG, Zhu JM, et al. Sun's procedure for chronic type A aortic dissection: total arch replacement using a tetrafurcate graft with stented elephant trunk implantation. Ann Cardiothorac Surg 2013;2:665-6.

14. Hibbert K, Rice M, Malhotra A. Obesity and ARDS. Chest 2012;142:785-90.

15. Sheng $W$, Yang HQ, Chi YF, et al. Independent risk factors for hypoxemia after surgery for acute aortic dissection. Saudi Med J 2015;36:940-6.

16. Ball L, Hemmes SNT, Serpa Neto A, et al. Intraoperative ventilation settings and their associations with

Cite this article as: Zhou J, Pan J, Yu Y, Huang W, Lai Y, Liang W, Nong L, Liu X, Chen S, Xu Y, He W, Xu Y, Liu X, Li Y, Huang Y, Sang L. Independent risk factors of hypoxemia in patients after surgery with acute type A aortic dissection. Ann Palliat Med 2021;10(7):7388-7397. doi: 10.21037/apm-21-1428 postoperative pulmonary complications in obese patients. Br J Anaesth 2018;121:899-908.

17. Pirrone M, Fisher D, Chipman D, et al. Recruitment Maneuvers and Positive End-Expiratory Pressure Titration in Morbidly Obese ICU Patients. Crit Care Med 2016;44:300-7.

18. Basile DP, Anderson MD, Sutton TA. Pathophysiology of acute kidney injury. Compr Physiol 2012;2:1303-53.

19. Liu J, Xue Y, Jiang W, et al. Thyroid Hormone Is Related to Postoperative AKI in Acute Type A Aortic Dissection. Front Endocrinol (Lausanne) 2020;11:588149.

20. Helgason D, Helgadottir S, Ahlsson A, et al. Acute Kidney Injury After Acute Repair of Type A Aortic Dissection. Ann Thorac Surg 2021;111:1292-8.

21. Shu S, Wang Y, Zheng M, et al. Hypoxia and HypoxiaInducible Factors in Kidney Injury and Repair. Cells 2019;8:207.

22. Schetz M, De Jong A, Deane AM, et al. Obesity in the critically ill: a narrative review. Intensive Care Med 2019;45:757-69. 